\title{
Evolution de la motivation scolaire des adolescents: différences selon la filière et le genre
}

\section{Philippe A. Genoud, Guillaume Ruiz et Jean-Luc Gurtner}

Cet article propose une analyse de l'évolution de la motivation d'adolescents en contexte scolaire. L'accent est mis sur les différences qui peuvent être observées dans cette évolution en fonction du genre et de la filière d'étude (regroupement selon le niveau de compétence) des élèves. Quatre dimensions de l'Echelle multidimensionnelle de motivation pour les apprentissages scolaires (EMMAS, Ntamakiliro, Monnard \& Gurtner, 2000) ont été testées auprès de 332 élèves des deux sexes et des trois degrés du cycle d'orientation (enseignement secondaire inférieur). Ces dimensions sont la volonté d'apprendre, l'attrait de l'école, le sentiment de compétence et l'anxiété face aux évaluations. Nos résultats montrent que si la motivation diminue globalement au cours des trois années de la scolarité secondaire quelle que soit la dimension considérée, elle s'avère manifestement différente selon la filière d'étude et selon le genre. Un effet d'interaction entre genre, degré et filière est également observé sur la dimension du sentiment de compétence. Ces résultats démontrent l'utilité de prendre en compte ces trois paramètres si l'on veut comprendre la motivation des élèves et la soutenir durant la phase critique de l'adolescence.

\section{Introduction}

Dans les recherches s'intéressant au fonctionnement de l'élève dans ses apprentissages, des mesures telles que celles concernant l'engagement ou la motivation sont très fréquentes. Selon les modèles proposés par nombre d'auteurs, la motivation semble en effet être une sorte de catalyseur permettant d'expliquer - indépendamment des caractéristiques liées aux compétences objectives de l'élève une part non négligeable de variance de la réussite scolaire. Nombreuses sont les recherches qui ont en effet mis en exergue les liens importants entre ces deux concepts, particulièrement durant la période de l'adolescence (voir par exemple Corbière, 1997; Gottfried, Fleming \& Gottfried, 2001). 
Alors que l'on observe un consensus relativement fort concernant l'impact de la motivation sur les performances scolaires, la définition de la motivation peine quant à elle à obtenir un compromis entre les chercheurs du domaine. Dans leur ouvrage, Vallerand et Thill (1993) montrent, en s'appuyant sur les différentes écoles de la psychologie, les diverses approches possibles pour aborder ce concept. Même si l'approche cognitive reste largement prédominante dans les multiples publications, le concept de motivation ne possède toutefois pas de définition commune admise par tous les spécialistes.

La caractéristique prédominante de la motivation dans le domaine scolaire reste alors l'aspect multifactoriel qu'on peut lui donner afin d'élargir le "noyau» que constitue l'engagement de l'élève dans les activités d'apprentissage à un spectre plus large prenant en compte les déterminants de cet engagement et d'autres aspects plus périphériques mais étroitement articulés avec les autres dimensions (Wigfield, Eccles \& Rodriguez, 1998), tel que cela apparaît par exemple dans la théorie du flow (Csikszentmihalyi, 2000). Outre la prise en compte de la volonté qu'a l'apprenant de vouloir s'investir dans le travail scolaire, on trouve, de façon récurrente dans les recherches, les dimensions appelées intérêt ou attrait des études. Les orientations ou buts motivationnels (buts d'apprentissage, de performance, sociaux, etc.) constituent également une gamme de dimensions qui ont, à elles seules, fait l'objet d'une attention toute particulière dans la littérature scientifique (Ames \& Archer, 1988; Barron \& Harackiewicz, 2001). Parmi les dimensions cognitives également, on ne saurait oublier les différents construits liés aux perceptions ou croyances du sujet tels que, par exemple, le sentiment de compétence ou de contrôlabilité (Malmberg \& Little, 2007; Pajares, 1996). On relèvera aussi l'aspect émotionnel que peuvent générer ces croyances, comme par exemple l'anxiété face aux évaluations. Un sentiment de compétence faible risque de générer des inquiétudes pour un examen futur et contribuer ainsi au désinvestissement de l'élève dans son travail. Ces dimensions s'avèrent donc étroitement liées entre elles; certaines recherches mettent en évidence les liens entre motivation et anxiété (Gottfried, 1985; Pekrun, Goetz, Titz \& Perry, 2002), variables considérées alors comme éléments dissociés, d'autres incluent l'anxiété comme une composante à part entière de la motivation (Pintrich \& De Groot, 1990). Plus récemment, de nombreux auteurs abordent la motivation avec des dimensions liées à la régulation des comportements (Bouffard, Bouchard, Goulet, Denoncourt \& Couture, 2005) et à la prise en compte d'aspects sociaux (Anderman \& Kaplan, 2008).

Quelles que soient les dimensions retenues dans les diverses recherches, il importe donc - en l'absence de consensus sur ce que recoupe la motivation - d'en préciser clairement les dimensions retenues. Avec la perspective essentiellement cognitive dans laquelle notre recherche s'inscrit, la définition donnée par Viau (1997) nous semble particulièrement pertinente: «État dynamique qui a ses origines dans les perceptions qu'un élève a de lui-même et de son environnement et qui l'incite à choisir une activité, à s'y engager et à persévérer dans son accom- 
plissement afin d'atteindre un but» (p. 7). Les dimensions sélectionnées pour cette étude (voir plus loin) s'inscrivent donc dans cette perspective multifactorielle.

\section{Perspective Longitudinale}

La motivation de l'élève n'est pas une caractéristique stable tel un facteur de personnalité. Durant son parcours scolaire, on peut observer des fluctuations plus ou moins importantes. Toutefois, malgré la dynamique individuelle qui influence ces changements de motivation (Galand \& Bourgeois, 2006), on retrouve - dans une perspective longitudinale - des schémas beaucoup plus généralisables. Par exemple, divers auteurs ont mis en évidence que les jeunes enfants montrent une certaine difficulté à distinguer effort et capacité (Folmer et al., 2008). Les élèves qui attribuent l'atteinte d'un objectif à une disposition innée ne vont pas s'engager de la même manière que leurs pairs qui la perçoivent comme dépendante de leur travail. Avec l'âge cependant, les élèves différencient de manière plus consistante les deux concepts. Au fur et à mesure de l'avancement dans la scolarité, la motivation intrinsèque des élèves se révèle de plus en plus stable (Gottfried, A. E., Fleming \& Gottfried, A. W., 2001) et il est donc plus aisé de prédire leur motivation pour les tâches scolaires. De plus, cette motivation va globalement décliner avec l'âge, et ceci depuis le début de la scolarité. Frey et Ruble (1987), tout comme dans d'autres recherches plus récentes (Bouffard, Marcoux, Vezeau \& Bordeleau, 2003) observent ce déclin dès le début de l'école primaire, même s'il ne touche pas toutes les matières au même moment.

Par la suite, cette diminution de la motivation va se poursuivre et souvent se généraliser à l'ensemble des disciplines scolaires. Elle s'explique habituellement par la transition de l'école primaire au secondaire, mais également par les changements liés au début de l'adolescence (Eccles \& Midgley, 1989). De nombreuses études confirment ce phénomène, tant pour l'engagement dans les tâches d'apprentissage que pour le sentiment de compétence (Eccles, Wiegfield, Harold \& Blumenfeld, 1993; Gurtner \& Genoud, 2006; Midgley, 1993). Cette chute progressive semble se stabiliser vers la fin de l'adolescence (14-15 ans selon Gurtner, Monnard \& Genoud, 2001, ou 16-17 ans selon Gottfried et al., 2001), période qui correspond généralement, de part et d'autre de l'Atlantique, à la fin de la scolarité obligatoire.

Ce pattern de variation de la motivation - qui est souligné par nombre de chercheurs - est bien évidemment une tendance générale, calculée en moyenne sur de larges échantillons. Elle ne touche cependant pas forcément chaque élève pris séparément. Dans notre recherche menée auprès d'adolescents de 11 à 16 ans, nous nous attendons donc à retrouver un déclin global de motivation au cours des trois années (méthode transversale), et formulons cette hypothèse tant pour la volonté d'apprendre que pour l'attrait des études et le sentiment de compétence des élèves. 


\section{Différences selon la filière}

Ce phénomène de déclin de motivation généralement observé durant la scolarité peut être accru ou atténué par d'autres facteurs. En effet, en fonction de changements contextuels particuliers, l'engagement et la persévérance pour le travail scolaire risquent de varier fortement. De nombreuses recherches mettent en évidence des aspects liés à l'influence des pairs (Nelson \& DeBacker, 2008), du climat de classe et de l'enseignant (Urdan \& Schoenfelder, 2006), des parents (Wentzel, 1998), etc. L'approche écologique a bien montré que les différents contextes dans lesquels l'élève évolue jouent un rôle considérable (Anderson, Hamilton \& Hattie, 2004; Gurtner et al., 2001).

Lors de la transition vers le secondaire, l'importance du changement de contexte s'avère être un aspect très important, tant au niveau du climat relationnel (Davis, 2006) que de la répartition des élèves - dans nombre de systèmes scolaires - en différentes filières en fonction de leurs compétences. On parle généralement de filière lorsqu'il s'agit d'un placement explicite de l'élève dans un groupe scolaire relativement homogène, placement basé sur ses résultats antérieurs et qui a un impact visible sur l'orientation future de l'élève (par exemple la possibilité d'avoir accès aux études supérieures ou non).

Les dispositifs scolaires basés sur les filières ont été - et sont encore - controversés. Un des avantages liés au fait d'avoir des groupes ayant des compétences relativement homogènes concerne la possibilité d'utiliser un matériel adapté qui est, par conséquent, plus susceptible de motiver les élèves. Mais on trouve également les effets d'assimilation et de contraste faisant varier le sentiment de compétence des apprenants. En Allemagne, où le système de filière est tangible, Trautwein et ses collègues ont pu souligner ces deux effets ayant des répercussions opposées (Trautwein, Lüdtke, Marsh, Köller \& Baumert, 2006). Selon ces auteurs, en contrôlant la variable que représente la performance scolaire, on peut voir apparaître un effet positif d'assimilation sur le sentiment de compétence des élèves des filières supérieures. Ces derniers ont alors le sentiment de faire partie des «bons» élèves puisqu'ils ont été regroupés ensemble et étiquetés comme étant les «meilleurs». Toutefois, cet effet d'assimilation est compensé par l'influence exercée par l'effet de contraste. Les élèves se retrouvent alors dans un groupe plus homogène (entre meilleurs), et les comparaisons aux autres leur sont par conséquent globalement moins favorables. Pour les élèves des filières inférieures, l'effet négatif de l'assimilation au groupe le plus faible (phénomène d'étiquetage) est contrebalancé avec le rétrécissement de l'étendue des comparaisons possibles à l'intérieur même du groupe, permettant ainsi d'augmenter le sentiment de compétence tout en diminuant l'anxiété (Wigfield, Eccles \& Rodriguez, 1998).

Les résultats contrastés des recherches menées dans ce domaine soulignent le manque de consistance des effets liés à cette répartition des élèves en filière (Berends, 1995; Bouffard \& Couture, 2003; Slavin, 1990). De plus, les différences observées sont souvent faibles à modérées et, par conséquent, ne permettent pas de tirer des conclusions très solides tant les autres éléments de contexte restent 
difficiles à contrôler. Sans pouvoir postuler des hypothèses orientées, nous nous proposons de mettre en évidence dans cette recherche - et sous une forme exploratoire - les différences entre les filières et d'interpréter les éventuels écarts observés.

\section{Différences selon le genre}

A l'instar du déclin de motivation observé tout au long de la scolarité, les différences observées selon le genre recueillent un fort consensus parmi les chercheurs. En laissant de côté les premières étapes préscolaires durant lesquelles on n'observe d'ordinaire que peu de différences de motivation (Patrick, Mantzicopoulos, Samarapungavan \& French, 2008), à l'adolescence, les filles disent généralement consacrer plus d'effort et de persistance que les garçons (Goodenow \& Grady, 1993; Ryan, 2001). Les causes de cette tendance sont certainement liées à des stéréotypes véhiculés par les styles parentaux (Heaven \& Newbury, 2004) ou de l'enseignant (Schaffner \& Schiefele, 2007).

Au niveau du sentiment de compétence, c'est alors les garçons qui obtiennent la plupart du temps des scores plus élevés (Pajares, 1996), ceci tant chez les préadolescents (Malmberg \& Little, 2007) que chez leurs pairs plus jeunes (Stetsenko, Little, Gordeeva, Grasshof \& Oettingen, 2000). Lauto-évaluation systématiquement plus faible des filles trouve à l'adolescence également un écho dans une anxiété plus élevée face au travail et aux évaluations scolaires (Marsh, Martin \& Cheng, 2008; Martin, 2004), et surtout une plus grande variété de niveaux d'anxiété selon les disciplines que chez les garçons (Wolters \& Pintrich, 1998). En effet, d'autres résultats suggèrent aussi que les filles se montrent non seulement plus critiques envers elles-mêmes (Frey \& Ruble, 1987), mais sont également plus précoces pour distinguer leur compétence et leur motivation en fonction des matières enseignées alors que les garçons réagissent de manière plus globale (Bouffard, Marcoux, Vezeau \& Bordeleau, 2003).

Toutefois, bien que les différences selon le genre soient plutôt faibles comparées aux influences du contexte (Dowson, McInerney \& Nelson, 2006), elles apparaissent systématiquement dans de nombreuses recherches. Nous faisons donc l'hypothèse que les filles feront état de plus hauts scores pour la volonté d'apprendre, l'attrait des études et l'anxiété que les garçons, alors que ces derniers auront un sentiment de compétence plus exacerbé.

\section{Différence selon la matière scolaire}

Les différences de motivation peuvent également se retrouver en fonction de la discipline scolaire abordée lors d'un cours. Certains élèves peuvent en effet s'investir fortement dans le cadre de l'apprentissage d'une matière qu'ils aiment et, à l'inverse, se désengager des travaux qui les intéressent moins. Différentes études (Goetz, Frenzel, Pekrun, Hall \& Lüdtke, 2007; Gottfried, 1985) analysent les différences entre matières scolaires en termes de motivation, de plaisir ou 
d'anxiété et décrivent des spécificités relatives aux disciplines (le domaine des mathématiques étant très fréquemment investigué).

Toutefois, bien que l'on ne puisse pas nier l'effet des matières scolaires, du contexte et des situations didactiques (rôle de l'enseignant, du type de tâche, etc.) sur la motivation des apprenants, l'impact de l'âge et du genre semble être suffisamment important pour envisager une généralisation des résultats quelle que soit la discipline concernée (Marsh, Martin \& Cheng, 2008). Des mesures plus "globales» de la motivation des élèves pour les apprentissages en général à l'école permettent en effet de repérer le rôle de certains facteurs individuels de façon plus consistante et au fil du temps.

\section{Méthodologie}

La recherche s'est déroulée dans un Cycle d'Orientation du canton de Fribourg, offrant une scolarité séparée en trois filières, à savoir Exigences de base (filière inférieure offrant la perspective d'un apprentissage), Générale (filière intermédiaire) et Prégymnasiale (filière supérieure donnant accès au secondaire II puis à l'Université). Les enseignants ayant une responsabilité de classe ont été sollicités pour participer à cette recherche et c'est donc sur une base volontaire que la sélection des classes a été effectuée. La procédure prévoyait deux mesures durant le premier trimestre de l'année 2007-2008 (début novembre et mi-décembre), délai permettant d'envisager d'éventuelles fluctuations de motivation scolaire chez les élèves.

\section{Echantillon}

Léchantillon regroupe les élèves de 17 classes, soit un nombre total de 332 sujets, répartis sur les trois années scolaires (149 en 1ère, 86 en 2ème, 97 en 3ème) et dans les trois filières (voir Tableau 1). Ces élèves ont donc complété, à deux reprises, un questionnaire permettant d'évaluer leur motivation pour le travail scolaire. Quelques-uns $(n=24)$ n'ont pu cependant participer qu'à une seule des deux passations. Le pourcentage de garçons s'élève à $54 \%$ (contre $46 \%$ de filles) et l'âge de ces élèves se situe entre 11 et 16 ans $(\mathrm{m}=13.7, \mathrm{~s}=1.1)$.

Tableau 1. Répartition garçons/filles dans les filières

\begin{tabular}{|lccc|}
\hline Filière & Garçons & Filles & Total \\
\hline Exigences de base & 67 & 39 & 106 \\
Générale & 57 & 57 & 114 \\
Prégymnasiale & 56 & 56 & 112 \\
\hline Total & 180 & 152 & 332 \\
\hline
\end{tabular}




\section{Instrument}

Le questionnaire utilisé est une adaptation de l'Echelle multidimensionnelle de motivation pour les apprentissages scolaires (EMMAS, Ntamakiliro, Monnard \& Gurtner, 2000). Cette dernière - constituée à la base de 7 dimensions - a été adaptée dans divers contextes tout en conservant des caractéristiques psychométriques très satisfaisantes (Schumacher, Genoud \& Gurtner, 2007). A l'instar de l'adaptation faite pour les classes du niveau secondaire II, le questionnaire réaménagé pour cette recherche a été limité à 4 dimensions (voir Tableau 2), sans distinguer les différentes disciplines scolaires (évaluation de la motivation pour les apprentissages de manière générale), ceci afin d'obtenir une mesure plus globale.

Le questionnaire comprend au final 20 affirmations (voir exemples dans le Tableau 2) auxquelles le répondant donne, à l'aide d'une échelle de Likert, un degré d'accord compris allant de 1 (= pas d'accord) à 7 (= d'accord). Les indices d'homogénéité interne, également présentés dans le tableau pour les deux passations indiquent des valeurs très satisfaisantes.

Tableau 2. Dimensions de l'échelle multidimensionnelle de motivation, avec exemples ditems et alpha de Cronbach pour chaque passation

\begin{tabular}{|llcc|}
\hline Dimensions & Exemple d'item & Alpha de Cronbach \\
\hline VA - Volonté d'apprendre & Je suis prêt-e à travailler dur pour mes cours. & .91 & .93 \\
SC - Sentiment de compétence & J'ai de bonnes aptitudes pour étudier. & .80 & .83 \\
AE - Attrait des études & J'ai du plaisir à étudier au CO. & .85 & .84 \\
EA - Etat d'anxiété & Je suis nerveux/se pendant les interrogations. & .86 & .86 \\
\hline
\end{tabular}

Les analyses en composantes principales effectuées séparément sur les deux passations laissent apparaître clairement les 4 facteurs postulés (selon le critère de Kaiser). Les indices KMO sont de .90 et .88 (tests de Bartlett significatifs) et les pourcentages de variance expliquée sont respectivement de $67 \%$ pour la première passation et de $68 \%$ pour la seconde. La comparaison des solutions factorielles mise en évidence par l'indice de Tucker (Lorenzo-Seva \& Ten Berge, 2006) montre une congruence très élevée $(\mathrm{ICmoyen}=.98)$.

\section{Présentation des résultats}

L'analyse des données recueillies a été effectuée en deux temps. Les données ont tout d'abord été soumises à des analyses de variance exploratoires permettant de mettre en évidence les effets principaux et les effets d'interactions sur les quatre dimensions de la motivation, ceci tant pour la première que pour la seconde passation. Afin d'améliorer la lisibilité des résultats présentés ci-dessous, nous avons opté pour un regroupement des données avec l'utilisation de tests séparés pour 
répondre à chaque question ou hypothèse spécifique. Toutefois, les résultats des analyses de variance multivariées indiquent des différences significatives pour les mêmes variables.

En ce qui concerne les deux mesures réalisées (prises avec un intervalle de 6 semaines), nous constatons une stabilité globale relativement forte. Les corrélations entre les deux temps de mesure sont plutôt élevées (moyenne de $r=.79$ pour les quatre dimensions) et indiquent ainsi une stabilité relative marquée. Ainsi, par exemple, les élèves les plus motivés à la première passation restent les plus motivés à la seconde. La stabilité de niveau est également élevée (en moyenne sur l'ensemble de l'échantillon). En effet, les tests t de Student appariés produisent des résultats non significatifs et la différence moyenne (absolue) entre les scores est inférieure à .03 (sur une échelle de 1 à 7 ), indiquant que - malgré de petites fluctuations individuelles - les mesures ne varient que très faiblement lorsqu'on considère l'ensemble des sujets.

Au niveau du genre, les analyses effectuées nous ont permis de mettre en évidence des différences entre filles et garçons. Même si ces différences ne sont pas très marquées pour certaines dimensions (voir Figure 1), elles sont cependant toutes significatives. Les filles indiquent une plus grande volonté d'apprendre ( $\mathrm{m}$ $=5.46, \mathrm{~s}=1.10 v \mathrm{~s}=5.02, \mathrm{~s}=1.22 ; \mathrm{t}(330)=3.43, \mathrm{p}<.01)$ et un plus fort at trait des études $(\mathrm{m}=5.26, \mathrm{~s}=1.17$ vs $\mathrm{m}=4.95, \mathrm{~s}=1.22 ; \mathrm{t}(330)=2.37, \mathrm{p}<.05)$ que les garçons. Ces derniers par contre se sentent plus compétents $(\mathrm{m}=5.05, \mathrm{~s}$ $=1.05 v s \mathrm{~m}=4.73, \mathrm{~s}=1.08 ; \mathrm{t}(330)=2.75, \mathrm{p}<.01)$ et, surtout, disent éprouver beaucoup moins d'anxiété face aux évaluations $(\mathrm{m}=3.50, \mathrm{~s}=1.35 \mathrm{vs} \mathrm{m}=4.56$, $\mathrm{s}=1.47 ; \mathrm{t}(330)=6.82, \mathrm{p}<.01)$. Ces différents résultats - dont les valeurs sont données ici sur la moyenne des deux passations - se retrouvent séparément dans chacune d'entre elles dans des proportions identiques.

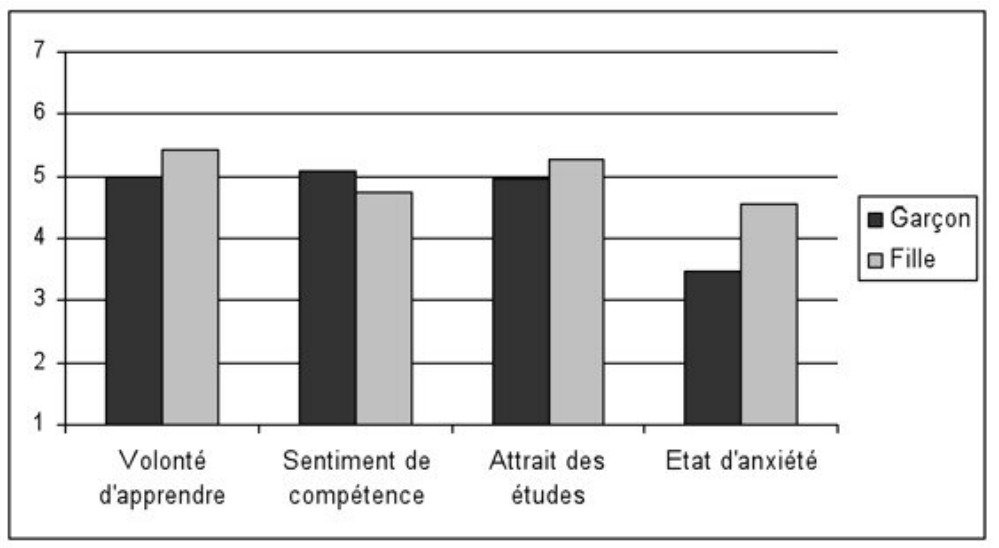

Figure 1. Différences garçons/filles sur les 4 dimensions de la motivation (moyenne des deux passations) 
Nous avons également testé, à l'aide d'analyses de variance à un facteur, l'effet de la filière d'étude sur chacune des dimensions de la motivation. L'effet est significatif sur une seule dimension, le sentiment de compétence $(\mathrm{F}(2,329)=$ 16.86, $\mathrm{p}<.01)$. Les résultats des tests post hoc de Tukey indiquent que les élèves de la section prégymnasiale - filière permettant l'accès au secondaire II - montrent des scores significativement plus élevés $(\mathrm{m}=5.34, \mathrm{~s}=1.04)$ que leurs camarades des deux autres sections (Générale: $\mathrm{m}=4.81, \mathrm{~s}=0.95$; Exigences de base: $m=4.55, s=1.10$ ). Pour les trois autres dimensions, on n'observe que de faibles différences (Figure 2).

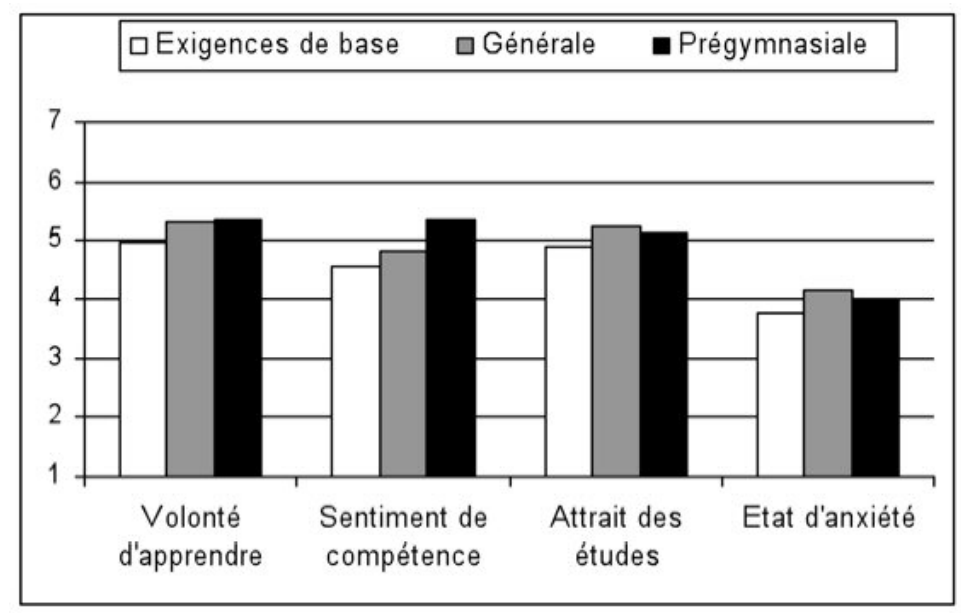

Figure 2. Différences selon la filière (trois degrés confondus)

Afin d'observer l'évolution de la motivation durant le parcours scolaire secondaire des élèves (trois dernières années de la scolarité obligatoire), nous avons construit des courbes présentant, pour les quatre dimensions, les fluctuations de motivation (Figure 3). Cette représentation correspond à un plan méthodologique transversal dans la mesure où ce ne sont pas les mêmes élèves qui ont été suivis sur les trois années, bien que nous ayons, pour chaque volée, deux passations dont nous avons calculé la moyenne. En ce qui concerne les statistiques inférentielles, nous avons réalisé des analyses de variance. Nos résultats indiquent un effet marqué des trois degrés scolaires sur la volonté d'apprendre $(\mathrm{F}(2,329)=$ $20.29, \mathrm{p}<.01$ ), variable pour laquelle les scores des trois groupes d'élèves different significativement les uns des autres $(\mathrm{p}<.01)$ selon les tests post hoc de Tukey (1ère année: $m=5.62, s=1.09$; 2ème année: $m=5.10, s=1.10$; 3ème année: $\mathrm{m}=4.71, s=1.19)$. Il en est de même pour l'attrait des études $(\mathrm{F}(2,329)=$ $29.15, \mathrm{p}<.01)$, avec également des différences significatives entre les trois degrés (1ère année: $m=5.58, s=1.03$; 2ème année: $m=4.90, s=1.23$; 3ème année: $m$ $=4.50, s=1.13)$. Ces deux dimensions présentent en effet un score d'un point 
plus faible en moyenne entre la 1ère et la 3ème année. L'état d'anxiété aussi évolue; il est globalement moins fort pour les élèves de 3ème année (toutes filières confondues) que pour les plus jeunes $(\mathrm{F}(2,329)=4.47, \mathrm{p}<.05)$. Les tests post hoc se révèlent significatifs ( $\mathrm{p}<.01)$, à l'exception de celui calculé entre les élèves de 2ème et de 3ème année (1ère année: $\mathrm{m}=4.19, \mathrm{~s}=1.49$; 2ème année: $\mathrm{m}=4.05$, $s=1.55$; 3ème année: $\mathrm{m}=3.62, s=1.42$ ). Le sentiment de compétence, par contre, ne varie que faiblement durant la scolarité secondaire, du moins sur cette représentation transversale, si on examine l'évolution les trois sections confondues (1ère année: $\mathrm{m}=5.05, s=1.02$; 2ème année: $\mathrm{m}=4.74, s=1$.10; 3ème année: $\mathrm{m}$ $=4.83, s=1.13)$.
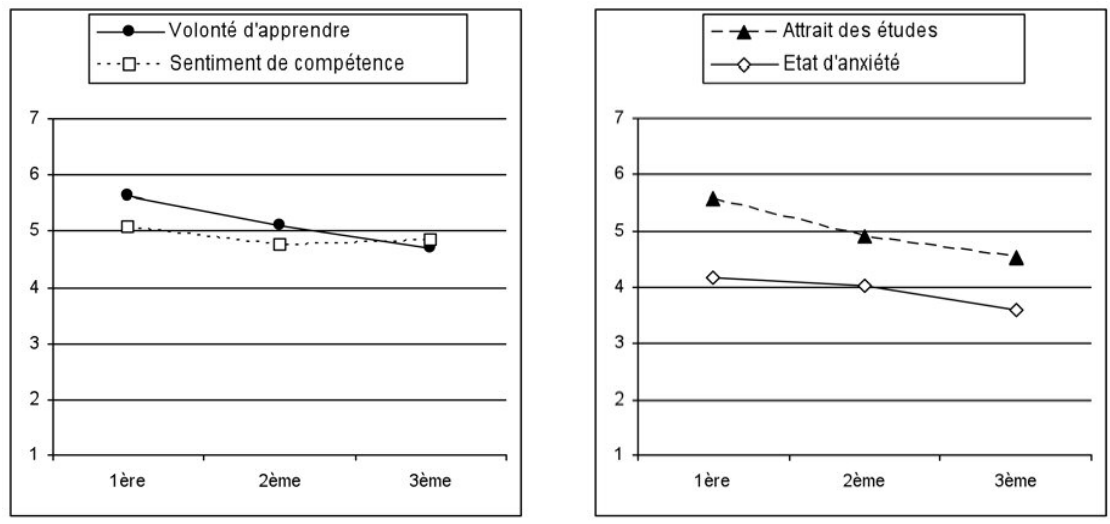

Figure 3. Evolution (plan transversal) de la motivation pour les différentes dimensions de la motivation

Ce dernier résultat concernant le sentiment de compétence peut être expliqué en partie par la présence d'évolutions différentes selon les filières. Le seul effet d'interaction significatif mis en évidence dans nos analyses $(\mathrm{F}(4,323)=3.86, \mathrm{p}<.01)$ concerne en effet cette variable. Si l'on observe (Figure 4) une diminution de ce sentiment de compétence au fil de la scolarité pour les élèves des sections prégymnasiale et exigences de base, cohérente avec ce qui a été souligné pour la volonté d'apprendre et l'attrait des études, les élèves de la section intermédiaire (générale) présentent par contre, vers la fin de leur scolarité, un sentiment de compétence supérieur à ce qu'il était en début de scolarité, presque identique à celui de leurs camarades de la section prégymnasiale. 


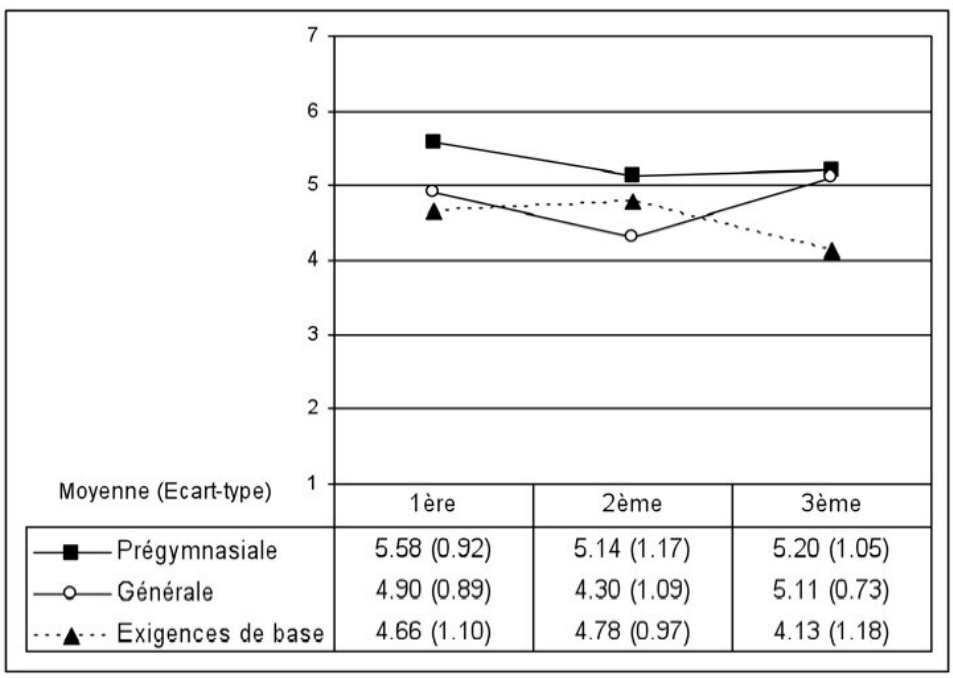

Figure 4. Evolution du sentiment de compétence selon la filière

\section{Discussion}

Dans cette recherche, nous nous sommes proposés de mesurer le niveau de motivation d'adolescents en contexte scolaire. Un exercice de ce type, bien que souvent pratiqué par les chercheurs, reste toujours pertinent puisqu'il permet non seulement d'affiner l'analyse des différentes dimensions constitutives de ce que l'on peut appeler motivation pour les apprentissages scolaires, mais également d'examiner de quelle manière elles évoluent, ici dans le contexte particulier de la scolarité secondaire organisée en trois filières d'étude.

Les différences observées en fonction du genre tout d'abord, semblent aller dans le sens de la plupart des études déjà menées sur le sujet (Martin, 2004; Ryan, 2001), et confirment ainsi nos hypothèses. Nous retrouvons en effet une différence significative en faveur des garçons pour le sentiment de compétence. Un des éléments qui semble pouvoir l'expliquer concerne spécifiquement les effets liés aux stéréotypes qui génèrent des croyances différentes concernant la réussite des filles et des garçons, mais également des attitudes inégales à leur encontre (Ruble \& Martin, 1998) qui risquent d'avoir des répercussions sur la perception de soi de ces derniers (Jussim \& Eccles, 1992). En effet, ce phénomène souligne bien le "poids des incitations et des injonctions, positives et négatives, des parents, des professeurs ou des condisciples, toujours prompts à rappeler de manière tacite ou explicite au destin qui leur est assigné» (Bourdieu, 1998, p. 130). A l'inverse, ces stéréotypes, ainsi qu'une maturité quelque peu plus précoce, pourraient faire apparaître un avantage sensible sur la volonté d'apprendre 
des filles. Ce résultat est également à mettre en relation avec un sentiment d'appartenance scolaire plus fort pour ces dernières (Goodenow \& Grady, 1993), tout comme un attrait des études plus marqué. L'écart le plus important concerne cependant l'état d'anxiété qui apparaît comme nettement plus présent chez les filles que chez les garçons, comme cela se retrouve du reste dans un grand nombre de recherches (Ollendick, Matson \& Helsel, 1985). Là encore, les attentes jouent probablement un rôle prépondérant. Harris et Ferrari (1983) indiquent à ce sujet que les parents seraient moins enclins, voire capables, de percevoir le comportement d'un garçon comme étant empreint d'anxiété. Ils évoquent également l'hypothèse que les adultes pourraient tolérer plus facilement de l'anxiété chez les filles. En plus de ce facteur socioculturel, des effets de désirabilité sociale sont aussi à souligner, les garçons étant peut-être moins prompts à reconnaître leurs inquiétudes face aux évaluations (Ollendick, 1983).

À l'instar d'autres recherches (Frey \& Ruble, 1987), nous avons constaté avec notre perspective transversale - une baisse de l'attrait des études et, corollairement, de la volonté d'apprendre au fil des trois années de scolarité secondaire. Ce constat, bien que préoccupant en soi, s'explique par trois raisons principales. En premier lieu, la période de l'adolescence est caractérisée par des changements physiques et cognitifs. Dans une revue de littérature très fournie, Anderman et Maehr (1994) relèvent que les adolescents discriminent mieux que leurs pairs plus jeunes l'effort de l'aptitude dans l'explication de la réussite scolaire. Faisant l'expérience d'une répartition en filières hiérarchisées selon l'aptitude, ils ressentent alors fortement l'impact de cette dernière sur leurs résultats et tendent donc à diminuer leur investissement dans les apprentissages scolaires. Deuxièmement, cette période est également caractérisée par le développement d'intérêts plus marqués dans des domaines extrascolaires, ceci au détriment de celui lié aux études. De plus, plusieurs études constatent un engagement de plus en plus diversifié selon la discipline scolaire (Eccles \& Midgley, 1989) indiquant alors une chute de motivation plus forte pour certaines matières jugées peu intéressantes. Finalement, alors que la transition de l'école primaire au secondaire a pu redonner à certains un regain momentané de motivation, au fil du temps, la volonté d'investir des efforts va s'émousser.

Si divers auteurs constatent également un déclin du sentiment de compétence à l'adolescence, nos résultats indiquent en moyenne (sur l'ensemble des élèves) une faible variation, mais en aucun cas une diminution significative durant la scolarité. Notre hypothèse concernant cette variable n'est par conséquent pas confirmée. Si la méthodologie transversale de notre recherche nous oblige à relativiser ce constat, d'autres éléments explicatifs peuvent être soulignés. Bien que le sentiment de compétence ne semble fluctuer que légèrement au cours des trois années (voir Figure 3), on constate en effet clairement des évolutions propres à chaque filière (Figure 4). Ceci est également mis en évidence par l'effet d'interaction significatif relevé pour cette variable. Pour les élèves de la filière prégymnasiale, on observe un déclin entre la première et la deuxième année, dont la 
pente est comparable à leurs pairs de générale. Se retrouvant alors parmi des élèves les plus compétents, on peut faire l'hypothèse plausible d'une influence négative (bien que modérée) du "big-fish-little-pond-effect» décrit par Marsh (1987), où la comparaison avec les autres élèves les plus forts va faire baisser leur sentiment de compétence. Pour les élèves de la filière exigences de base, la tendance est inversée; ils partent avec le sentiment de compétence le plus faible mais, isolés des autres élèves, l'effet de contraste dommageable va s'atténuer. Avec la perspective de la fin de la scolarité, la situation change en troisième année. Les élèves de prégymnasiale restent relativement stables; pour la plupart d'entre eux, la poursuite vers la formation secondaire II (ou une autre formation) est souvent considérée comme un "acquis» à ce moment-là. L'ouverture vers un éventail important d'opportunités sur le plan du futur professionnel semble alors compenser l'éventuelle diminution qui serait associée à la baisse des autres indicateurs (comme la volonté d'apprendre ou l'attrait des études). Le constat est plus sombre pour les élèves en exigences de base. Leur sentiment de compétence (tout comme leur volonté d'apprendre et leur attrait pour les études) chute en troisième année. L'effet de comparaison créé par les filières ainsi que - et surtout - la difficulté pour un grand nombre d'entre eux de pouvoir envisager clairement l'après école obligatoire deviendrait alors prédominant.

Les élèves de la filière générale ne perçoivent pas leur situation de façon similaire à leurs pairs des deux autres filières. Après la séparation en filière (au début de la scolarité secondaire), on observe chez eux un sentiment de compétence proche de leurs pairs en exigences de base. Ils s'assimilent donc à ceux qui n'auront pas accès aux études supérieures, faisant ainsi ressortir le contraste avec leurs pairs de prégymnasiale. Vers la fin du cursus secondaire, une comparaison valorisante se dessine, ceci au moment où les élèves de cette filière générale peuvent entrevoir des perspectives professionnelles, comme l'obtention d'une place de stage. En ce qui concerne leur score moyen de sentiment de compétence, ils s'assimilent alors à leurs pairs de prégymnasiale, se différenciant alors clairement de leurs camarades en exigences de base.

Avec cette méthodologie transversale, l'effet significatif de la filière d'étude que nous avons observée sur le sentiment de compétence moyen prend une perspective différente. S'il apparaît globalement que plus les adolescents se situent dans une filière supérieure plus ils se sentent compétents, l'évolution de ce sentiment ne va pas être identique pour chacun. Alors que les facteurs contextuels presque inhérents à la scolarité secondaire (tels que l'augmentation de la dépersonnalisation et de la compétition ou la diminution de la qualité des relations avec les enseignants) sont mis en évidence dans la majorité des recherches relatives à ce domaine (Ferguson \& Fraser, 1999; Hirsch \& Rapkin, 1987), les événements contextuels différentiels sont plus rarement soulignés. Les constatations touchant l'ensemble des élèves - bien que récurrentes dans la littérature scientifique - ne sont donc pas à même d'expliquer les changements constatés dans notre étude. Nous observons au contraire ici que des perspectives futures différen- 
tes à l'approche de la fin de la scolarité obligatoire influenceront de manière distincte les élèves des trois sections.

\section{Conclusion}

Les divers résultats présentés dans cet article confirment dans leur ensemble les constatations faites par d'autres chercheurs au sujet de la motivation scolaire des adolescents. Que ce soit selon le genre ou selon les filières, les différences observées dans le contexte suisse romand sont globalement comparables à celles dégagées dans d'autres publications tant francophones qu'anglophones.

Cependant, en analysant l'évolution de la motivation plus en détail, on peut remarquer que les constatations très générales ne s'appliquent plus forcément à des groupes particuliers d'élèves voire même à des individus pris isolément. L'analyse de cette évolution selon les filières permet en effet de mettre à jour que le contexte n'est pas a priori le même pour tous les étudiants. La répartition en filières apporte certes des effets de contraste dans le sentiment de compétence des élèves, mais crée surtout des conditions fortement différentes. Une prise en compte des éléments contextuels dans les parcours scolaires individuels serait à même de confirmer l'impact de ces événements (positifs ou négatifs) sur le sentiment de compétence. Alors que le constat répétitif du déclin de la motivation dans des recherches très variées (tant sur le plan des caractéristiques de l'échantillon que de la méthodologie utilisée) pourrait pousser au fatalisme, un examen plus détaillé des différents contextes suggère des possibilités d'y trouver des pistes de remédiation. Les événements qui interviennent durant le cursus scolaire, même s'ils sont externes à celui-ci (comme une réponse positive concernant une place de stage) pourraient être à même d'avoir un impact marqué sur le sentiment de compétence. La référence sur laquelle se base un élève pour évaluer son sentiment de compétence varie, mais de façon non linéaire, et les recherches devraient pouvoir inclure des mesures plus fréquentes durant l'année afin d'évaluer des fluctuations de motivation plus sensibles qui pourraient ainsi être mises en lien avec des événements contextuels très ciblés dans le temps. La prise en compte du vécu individuel de chaque élève et sa mise en relation avec l'évolution des différentes dimensions de la motivation de celui-ci devraient ainsi permettre de repérer ces "leviers» générateurs de motivation, dont les enseignants pourraient tirer profit pour soutenir la motivation de chacun de leurs élèves individuellement. 


\section{Bibliographie}

Ames, C. \& Archer, J. (1988). Achievement goals in the classroom: students' learning strategies and motivation processes. Journal of Educational Psychology, 80 (3), 260-267.

Anderman, E. M. \& Maehr, M. L. (1994). Motivation and schooling in the middle grades. Review of Educational Research, 64 (2), 287-309.

Anderman, L. H. \& Kaplan, A. (2008). The role of interpersonal relationships in student motivation: Introduction to the special issue. Journal of Experimental Education, 76 (2), 115 119.

Anderson, A., Hamilton, R. J. \& Hattie, J. (2004). Classroom climate and motivated behaviour in secondary schools. Learning Environments Research, 7 (3), 211-225.

Barron, K. E. \& Harackiewicz, J. M. (2001). Achievement goals and optimal motivation: testing multiple goal models. Journal of Personality and Social Psychology, 80 (5), 706-722.

Berends, M. (1995). Educational stratification and students' social bonding to school. British Journal of Sociology of Education, 16 (3), 327-351.

Bouffard, T., Bouchard, M., Goulet, G., Denoncourt, I. \& Couture, N. (2005). Influence of achievement goals and self-efficacy on students' self-regulation and performance. International Journal of Psychology, 40 (6), 373-384.

Bouffard, T. \& Couture, N. (2003). Motivational profile and academic achievement among students enrolled in different schooling tracks. Educational Studies, 29 (1), 20-38.

Bouffard, T., Marcoux, M.-F., Vezeau, C. \& Bordeleau, L. (2003). Changes in self-perceptions of competence and intrinsic motivation among elementary schoolchildren. British Journal of Educational Psychology, 73 (2), 171-186.

Bourdieu, P. (1998). La domination masculine. Paris: Seuil.

Corbière, M. (1997). Une approche multidimensionnelle de la prédiction de la réussite scolaire. Orientation Scolaire et Professionnelle, 26 (1), 109-135.

Csikszentmihalyi, M. (2000). Flow. In A.E. Kazdin (Éd.), Encyclopedia of psychology (vol. 3, pp. 381-382). Washington, DC: American Psychological Association.

Davis, H. A. (2006). Exploring the contexts of relationship quality between middle school students and teachers. The Elementary School Journal, 106 (3), 193-223.

Dowson, M., McInerney, D. M. \& Nelson, G. F. (2006). An investigation of the effects of school context and sex differences on students' motivational goal orientations. Educational Psychology, 26 (6), 781-811.

Eccles, J. S. \& Midgley, C. (1989). Stage-environment fit: developmentally appropriate classrooms for young adolescents. In C. Ames \& R. Ames (Éd.), Research on motivation in education: Goals and cognition (vol. 3, pp. 139-186). San Diego: Academic Press.

Eccles, J. S., Wigfield, A., Harold, R. D. \& Blumenfeld, P. (1993). Age and gender differences in children's self- and task perceptions during elementary school. Child Development, 64 (3), 830-847.

Ferguson, P. D. \& Fraser, B. J. (1999). Changes in learning environment during the transition from primary to secondary school. Learning Environments Research, 1 (3), 369-383.

Folmer, A. S., Cole, D. A., Sigal, A. B., Benbow, L. D., Satterwhite, L. F., Swygert, K. E. et al. (2008). Age-related changes in children's understanding of effort and ability: Implications for attribution theory and motivation. Journal of Experimental Child Psychology, 99 (2), 114-134.

Frey, K. S. \& Ruble, D. N. (1987). What children say about classroom performance: sex and grade differences in perceived competence. Child Development, 58 (4), 1066-1078.

Galand, B. \& Bourgeois, E. (2006). (Se) motiver à apprendre. Paris: Presses Universitaires de France.

Goetz, T., Frenzel, A. C., Pekrun, R., Hall, N. C. \& Lüdtke, O. (2007). Between- and withindomain relations of students' academic emotions. Journal of Educational Psychology, 99 (4), 715-733. 
Goodenow, C. \& Grady, K. E. (1993). The relationship of school belonging and friends' values to academic motivation among urban adolescent students. Journal of Experimental Education, 62 (1), 60-71.

Gottfried, A. E. (1985). Academic intrinsic motivation in elementary and junior high school students. Journal of Educational Psychology, 77 (6), 631-645.

Gottfried, A. E., Fleming, J. S. \& Gottfried, A. W. (2001). Continuity of academic intrinsic motivation from childhood through late adolescence: A longitudinal study. Journal of Educational Psychology, 93 (1), 3-13.

Gurtner, J.-L. \& Genoud, P. A. (2006). Facteurs contextuels dans l'évolution de la motivation pour le travail scolaire au cours de l'adolescence. In B. Galand \& E. Bourgeois (Éd.), (Se) motiver à apprendre (pp. 115-124). Paris: Presses Universitaires de France.

Gurtner, J.-L., Monnard, I. \& Genoud, P. A. (2001). Towards a multilayer model of context and its impact on motivation. In S. Volet \& S. Järvelä (Éd.), Motivation in learning contexts: Theorical advances and methodological implications (pp. 189-208). Oxford: Pergamon.

Harris, S. L. \& Ferrari, M. (1983). Developmental factors in child behavior therapy. Behavior Therapy, 14 (1), 54-72.

Heaven, P. C. \& Newbury, K. (2004). Relationships between adolescent and parental characteristics, and adolescents' attitudes to school and self-rated academic performance. Australian Journal of Psychology, 56 (3), 173-180.

Hirsch, B.J. \& Rapkin, B.D. (1987). The transition to junior high school: a longitudinal study of self-esteem, psychological symptomatology, school life, and social support. Child Development, 58 (5), 1235-1243.

Jussim, L. \& Eccles, J. S. (1992). Teacher expectations II: Construction and reflection of student achievement. Journal of Personality and Social Psychology, 63 (6), 947-961.

Lorenzo-Seva, U. \& Ten Berge, J. M. (2006). Tucker's Congruence Coefficient as a meaningful index of factor similarity. Methodology: European Journal of Research Methods for the Behavioral and Social Sciences, 2 (2), 57-64.

Malmberg, L.-E. \& Little, T. D. (2007). Profiles of ability, effort, and difficulty: Relationships with worldviews, motivation and adjustment. Learning and Instruction, 17 (6), 739-754.

Marsh, H.W. (1987). The big-fish-little-pond-effect on academic self-concept. Journal of Educational Psychology, 79(3), 280-295.

Marsh, H. W., Martin, A. J. \& Cheng, J. H. (2008). A multilevel perspective on gender in classroom motivation and climate: Potential benefits of male teachers for boys? Journal of Educational Psychology, 100 (1), 78-95.

Martin, A. J. (2004). School motivation of boys and girls: Differences of degree, differences of kind, or both? Australian Journal of Psychology, 56 (3), 133-146.

Midgley, C. (1993). Motivation and middle level schools. In M.L. Maehr \& P.R. Pintrich (Éd.), Advances in motivation and achievement: Motivation and adolescent development (Vol. 8, pp. 217-274). Greenwich: JAI Press.

Nelson, R. M. \& DeBacker, T. K. (2008). Achievement motivation in adolescents: The role of peer climate and best friends. Journal of Experimental Education, 76 (2), 170-189.

Ntamakiliro, L., Monnard, I. \& Gurtner, J.-L. (2000). Mesure de la motivation scolaire des adolescents: construction et validation de trois échelles complémentaires. Orientation Scolaire et Professionnelle, 29 (4), 673-693.

Ollendick, T. H. (1983). Reliability and validity of the Revised Fear Survey Schedule for Children (FSSC-R). Behaviour Research and Therapy, 21 (6), 685-692.

Ollendick, T. H., Matson, J. L. \& Helsel, W. J. (1985). Fears in children and adolescents: Normative data. Behaviour Research and Therapy, 23 (4), 465-467.

Pajares, F. (1996). Self-efficacy beliefs in academic settings. Review of Educational Research, 66 (4), 543-578. 
Patrick, H., Mantzicopoulos, P., Samarapungavan, A. \& French, B. F. (2008). Patterns of young children's motivation for science and teacher-child relationships. Journal of Experimental Education, 76 (2), 121-144.

Pekrun, R., Goetz, T., Titz, W. \& Perry, R. P. (2002). Academic emotions in students' self-regulated learning and achievement: A program of qualitative and quantitative research. Educational Psychologist, 37 (2), 91-105.

Pintrich, P. R. \& De Groot, E. V. (1990). Motivational and self-regulated learning components of classroom academic performance. Journal of Educational Psychology, 82 (1), 33-40.

Ruble, D. N. \& Martin, C. L. (1998). Gender development. In W. Damon \& N. Eisenberg (Éd.), Handbook of child psychology: Social, emotional, and personality development (5e éd., vol. 3, pp. 933-1016). New York: John Wiley \& Sons.

Ryan, A. M. (2001). The peer group as a context for the development of young adolescent motivation and achievement. Child Development, 72 (4), 1135-1150.

Schaffner, E. \& Schiefele, U. (2007). The effect of experimental manipulation of student motivation on the situational representation of text. Learning and Instruction, 17 (6), 755772.

Schumacher, J. A., Genoud, P. A. \& Gurtner, J.-L. (2007, septembre). Measuring motivation: Adaptation of a questionnaire in diverse settings and perspectives for future research. Paper presented at the annual conference of the SSRE (Société Suisse de Recherche en Éducation) and the SSFE (Société Suisse pour la Formation des Enseignantes et des Enseignants), Kreuzlingen.

Slavin, R. E. (1990). Achievement effects of ability grouping in secondary schools: A best-evidence synthesis. Review of Educational Research, 60 (3), 471-499.

Stetsenko, A., Little, T. D., Gordeeva, T., Grasshof, M. \& Oettingen, G. (2000). Gender effects in children's beliefs about school performance: A cross-cultural study. Child Development, 71 (2), 517-527.

Trautwein, U., Lüdtke, O., Marsh, H. W., Köller, O. \& Baumert, J. (2006). Tracking, grading, and student motivation: Using group composition and status to predict self-concept and interest in ninth grade mathematics. Journal of Educational Psychology, 98 (4), 788806.

Urdan, T. \& Schoenfelder, E. (2006). Classroom effects on student motivation: goal structures, social relationships, and competence beliefs. Journal of School Psychology, 44 (5), 331-349.

Vallerand, R. J. \& Thill, E. E. (1993). Introduction à la psychologie de la motivation. Laval: Éditions Études Vivantes.

Viau, R. (1997). La motivation en contexte scolaire. Bruxelles: De Boeck \& Larcier.

Wentzel, K. R. (1998). Social relationships and motivation in middle school: the role of parents, teachers and peers. Journal of Educational Psychology, 90 (2), 202-209.

Wigfield, A., Eccles, J. S. \& Rodriguez, D. (1998). The development of children's motivation in school contexts. Review of Research in Education, 23, 73-118.

Wolters, C. A. \& Pintrich, P. R. (1998). Contextual differences in student motivation and self-regulated learning in mathematics, english, and social studies classrooms. Instructional Science, 26, 27-47.

Mots clés: Motivation, adolescence, filière d'étude, genre. 


\section{Entwicklung der schulischen Motivation von Jugendlichen: Differenzen aufgrund von Studiengängen und Geschlecht}

\section{Zusammenfassung}

Dieser Artikel befasst sich mit der Entwicklung der Lernmotivation von Schülerinnen und Schülern an der Sekundarschule. Vergleicht wird die Motivation von 180 Jungen und 152 Mädchen aller drei Schuljahre und aller Studiengänge. Gemessene Dimensionen sind die Bereitschaft zum Lernen, die Kompetenzeinschätzung, die Schulattraktivität und die Prüfungsangst, nach Ntamakiliro, Monnard und Gurtner (2000). Die Resultate zeigen, dass sich signifikante Unterschiede in der Entwicklung der Motivation nach Geschlecht und schulischen Profilen ergeben. Zudem zeigt sich eine Interaktion zwischen Studiengang/Profil und Schuljahr in der Entwicklung der Kompetenzeinschätzung. Diese Resultate beweisen wie wichtig es ist, Parameter wie Geschlecht, Studiengang/Profile oder Schuljahr in Betracht zu ziehen, um die Motivation der Jungen und Mädchen im Jugendalter zu unterstützen.

Schlagworte: Schulmotivation, Jugendalter, Geschlecht, Schulprofile, Studiengänge.

\section{L'evoluzione della motivazione scolastica degli adolescenti: differenze in funzione del percorso formativo e del genere}

\section{Riassunto}

L'articolo propone un'analisi dell'evoluzione della motivazione degli adolescenti nel contesto scolastico. L'accento è messo sulle differenze che si possono osservare in funzione del genere e del percorso formativo degli allievi. Quattro dimensioni della scala multidimensionale della motivazione per gli apprendimenti scolastici (EMMAS, Ntamakiliro, Monnard \& Gurtner, 2000) sono stati testati presso 332 allievi dei due sessi e dei tre livelli del ciclo d'orientamento (secondario I). Queste dimensioni sono la volontà di apprendere, l'interesse per la scuola, il sentimento di competenza e l'ansia delle valutazioni. I nostri risultati mostrano che, se la motivazione diminuisce globalmente nel corso dei tre anni della scolarità secondaria in tutte le dimensioni considerate, differenze manifeste appaiono in funzione del percorso formativo e del genere. Si osserva inoltre un effetto d'interazione tra genere, grado e filiera sulla dimensione del sentimento di competenza. Questi risultati mostrano l'utilità di prendere in considerazione i tre parametri se si vuole comprendere la motivazione degli allievi e sostenerli durante la fase critica dell'adolescenza.

Parole chiave: Motivazione, adolescenze, filiera di studio, genere 


\title{
Evolution of adolescents' school motivation: differences related to school track and gender
}

\begin{abstract}
This article presents an analysis of the evolution of adolescents' motivation within the school context. Comparisons are made according to gender and schooltracks. The study was carried out with 332 pupils of grade 7 through 9 who were asked to posit themselves on four dimensions of the Multidimensional scale of motivation for the school context (EMMAS, Ntamakiliro, Monnard \& Gurtner, 2000), namely learning intention, subjective competence, school attractiveness and test anxiety. Our results show that if motivation globally declines over the three years, differences in these evolutions appear according to gender and track. An interaction effect between these three variables can even be seen regarding subjective competence. These results confirm the importance of taking into account these three parameters in order to understand pupils' motivation and to sustain it during the critical phase of adolescence.
\end{abstract}

Key words: Motivation, adolescent, tracking (educational stratification), gender differences. 